\title{
BANACH ALGEBRAS OF OPERATOR SEQUENCES
}

\author{
M. SEIDEL AND B. SiLbermanN
}

Abstract. During the last decades it turned out to be fruitful to apply certain Banach algebra techniques in the theory of approximation of operators by matrix sequences. Here we discuss the case of operator sequences (acting on infinite dimensional Banach spaces and which do not necessarily converge strongly) and we derive analogous results concerning the stability and Fredholm properties of such sequences. For this, the notions of $\mathscr{P}$-Fredholmness and $\mathscr{P}$-strong convergence play an important role and are extensively studied. As an application we consider the finite sections of band-dominated operators on $l^{p}$-spaces, including the cases $p \in\{1, \infty\}$.

Mathematics subject classification (2010): 65J10, 47A58, 47B36, 47N40.

Keywords and phrases: Operator sequence, Fredholm sequence, approximation numbers, band-dominated operator, finite section method.

\section{REFERENCES}

[1] A. BÖTTCHER, On the approximation numbers of large Toeplitz operators, Documenta Mathematica 2 (1997), 1-29.

[2] A. Böttcher, B. Silbermann, Analysis of Toeplitz Operators, Second edition, Springer-Verlag, Berlin, 2006.

[3] S. N. Chandler-Wilde, M. Lindner, Limit Operators, Collective Compactness and the Spectral Theory of Infinite Matrices, Mem. Amer. Math. Soc. 210 (2011), No. 989.

[4] S. N. CHANDLER-WILDE, M. LINDNER, Boundary integral equations on unbounded rough surfaces: Fredholmness and the Finite Section Method, J. Integral Equations Appl. 20 (2008), 13-48.

[5] D. E. Edmunds, W. D. Evans, Spectral Theory and Differential Operators, Oxford University Press, New York, 1987.

[6] I. C. Gohberg, I. A. Feldman, Convolution equations and projection methods for their solution, Nauka, Moscow 1971; Engl. transl.: Amer. Math. Soc. Transl. of Math. Monographs 41, Providence, R. I., 1974; German transl.: Akademie-Verlag, Berlin 1974.

[7] I. C. Gohberg, N. Krupnik, One-dimensional Linear Singular Integral Equations, Birkhäuser Verlag, Basel, Boston, Berlin, 1992.

[8] R. Hagen, S. Roch, B. Silbermann, $C^{*}$-Algebras and Numerical Analysis, Marcel Dekker, Inc., New York, Basel, 2001.

[9] R. Hagen, S. Roch, B. Silbermann, Spectral Theory of Approximation Methods for Convolution Equations, Operator Theory: Advances and Applications, Vol. 74, Birkhäuser Verlag, Basel, 1995.

[10] G. Heinig, F. Hellinger, The finite section method for Moore-Penrose inversion of Toeplitz operators, Integral Equations Oper. Theory 19, 4 (1994), 419-446.

[11] P. Junghanns, S. Roch, B. Silbermann, Collocation methods for systems of Cauchy singular integral equations on an interval, Comp. Techn. 6 (2001), 88-124.

[12] A. V. KoZAK, A local principle in the theory of projection methods, Dokl. Akad. Nauk SSSR 212, 6 (1973), 1287-1289; Engl. transl.: Sov. Math. Dokl. 14, 5 (1974), 1580-1583.

[13] B. V. Lange, V. S. Rabinovich, On the Noether property of multidimensional discrete convolutions, Mat. Zametki 37, 3 (1985), 407-421.

[14] M. Lindner, The finite section method and stable subsequences, Appl. Num. Math. 60, 4 (2010), $501-512$.

[15] M. Lindner, Infinite Matrices and their Finite Sections, Birkhäuser Verlag, Basel, Boston, Berlin, 2006. 
[16] M. Lindner, Limit Operators and Applications on the Space of Essentially Bounded Functions, Dissertationsschrift, Chemnitz, 2003.

[17] H. Mascarenhas, B. Silbermann, Spectral approximation and index for convolution type operators on cones on $L^{p}\left(\mathbb{R}^{2}\right)$, Integral Equations Oper. Theory 65, 3 (2009), 415-448.

[18] A. PIETSCH, History of Banach spaces and linear operators, Birkhäuser Boston, Inc., Boston, MA, 2007.

[19] A. Pietsch, Operator Ideals, Verlag der Wissenschaften, Berlin, 1978.

[20] S. Prössdorf, B. Silbermann, Numerical Analysis for Integral and Related Operator Equations, Birkhäuser Verlag, Basel, Boston, Berlin, 1991.

[21] V. S. RABINOVICH, S. Roch, The Fredholm index of locally compact band-dominated operators on $L^{p}(\mathbb{R})$, Integral Equations Oper. Theory 57 (2007), 263-281.

[22] V. S. Rabinovich, S. Roch, J. RoE, Fredholm indices of band-dominated operators, Integral Equations Oper. Theory 49, 2 (2004), 221-238.

[23] V. S. Rabinovich, S. Roch, B. Silbermann, Band-dominated operators with operator-valued coefficients, their Fredholm properties and finite sections, Integral Equations Oper. Theory 40, 3 (2001), 342-381.

[24] V. S. Rabinovich, S. Roch, B. Silbermann, Fredholm theory and finite section method for band-dominated operators, Integral Equations Oper. Theory 30, 4 (1998), 452-495.

[25] V. S. Rabinovich, S. Roch, B. Silbermann, Limit Operators and Their Applications in Operator Theory, Birkhäuser Verlag, Basel, Boston, Berlin, 2004.

[26] V. S. Rabinovich, S. Roch, B. Silbermann, On finite sections of band-dominated operators, Operator Theory: Advances and Applications 181 (2008), 385-391.

[27] V. RAKoČEvić, J. ZEMÁNEK, Lower s-numbers and their asymptotic behaviour, Studia Math. 91, 3 (1988), 231-239.

[28] M. Reed, B. Simon, Methods of Modern Mathematical Physics IV: Analysis of Operators, Academic Press, New York, 1978.

[29] S. Roch, Finite Sections of Band-Dominated Operators, Memoirs of the AMS 191 (2008), No. 895.

[30] S. Roch, B. Silbermann, Non-strongly converging approximation methods, Demonstr. Math. 22, 3 (1989), 651-676.

[31] A. Rogozhin, B. Silbermann, Approximation numbers for the finite sections of Toeplitz operators with piecewise continuous symbols, J. Funct. Anal. 237, 1 (2006), 135-149.

[32] A. Rogozhin, B. Silbermann, Banach algebras of operator sequences: Approximation numbers, J. Oper. Theory 57, 2 (2007), 325-346.

[33] M. Seidel, B. Silbermann, Finite sections of band-dominated operators: $l^{p}$-theory, Complex Analysis and Operator Theory 2 (2008), 683-699.

[34] M. SeIdel, B. Silbermann, Banach algebras of structured matrix sequences, Lin. Alg. Appl. 430 (2009), 1243-1281.

[35] I. B. Simonenko, On multidimensional discrete convolutions, Mat. Issled. 3, 1 (1968), 108-127 (Russian). 